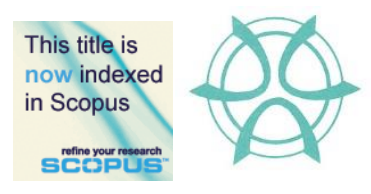

PLANNING MALAYSIA:

Journal of the Malaysian Institute of Planners

VOLUME 15 ISSUE 2 (2017), Page 75 - 84

\title{
HUMAN INTERACTION IN URBAN SPACES: A QUANTITATIVE ANAL YSIS IN URBAN PARK, SHAH ALAM CITY, SELANGOR
}

\author{
Filzani Illia Ibrahim¹, Dasimah Omar², \& Nik Hanita Nik Mohamad ${ }^{3}$ \\ ${ }^{1,2,3}$ Faculty of Architecture, Planning \& Surveying \\ UNIVERSITI TEKNOLOGI MARA (UiTM)
}

\begin{abstract}
This paper aims to provide valuable insight of the various dimensions of human needs' towards open spaces. This study employed a mixed method research design involving both quantitative and qualitative methods that were utilised to identify and evaluate the human-human interaction and human-nature interaction in the area of study. Primary data was gathered by using questionnaire survey that was administered to 861 respondents who were visitors to public parks. The finding of this study is intended to show the main domains that reflect human needs' toward open spaces together with perceived benefits to the open space users.
\end{abstract}

Keywords: human-human interaction, human-nature interaction, open spaces.

Date Received: $26^{\text {th }}$ July 2016

Date of Acceptance: $6^{\text {th }}$ July 2017 
Filzani Illia Ibrahim, Dasimah Omar, \& Nik Hanita Nik Mohamad

Human Interaction in Urban Spaces: A Quantitative Analysis in Urban Park, Shah Alam City, Selangor

\section{INTRODUCTION}

Historically, mankind has created open spaces for various reasons. Thus, open spaces are often viewed as the products of a complex society with different societal needs, interest and aesthetics, which evolved over time. Humans' relationship with open spaces is becoming increasingly complex due to the heterogeneous cultural and demographic dimensions of socio-economic, gender, type of activities and open space facilities. Hence, the growing scarcity of open spaces is of concern to the local authorities as there is a drastic reduction in good quality open spaces left in the urban areas.

The objectives of this paper are to identify the motives of urban dwellers in visiting open spaces, and to examine the human-human interaction and humannature interaction in open spaces. This study employed a mixed method research design involving both quantitative and qualitative methods that were utilised to identify and investigate the human-human interaction and human-nature interaction in the area of study. Primary data was gathered by using questionnaire survey that was administered to 861 respondents who were visitors to public parks in Shah Alam. The findings of this study show the main domains that reflect human needs towards open spaces. It is hoped that the findings of this study would assist landscape planners and designers in understanding the preferred conducive composition and configuration of human needs towards open spaces.

\section{HUMAN INTERACTION IN OPEN SPACES}

Open space act as a positive channel for people engagement and interaction (Omar, Illia \& Hanita, 2015). However, people value open space differently. According to Mutiara \& Isami (2012), urbanites prefer their open spaces with a variety of facilities for recreational activities instead of only attractive natural areas. Meanwhile, Machabee, Oleson and Kinzig (2004) claim that socioeconomic factor also determine the usage of open space, as residents of highincome neighbourhood were found to use their open spaces more than residents in the middle or low-income neighbourhoods.

According to Rasidi, Jamirsah and Said (2012), there is an increasing trend of research on the significance of open spaces. The development over the recent decades has resulted in the loss of forest, farm, forest fringe and other open space lands that somehow contributed to urban residents' quality of life. The growing scarcity of open space is of concern for local authorities since there is an alarming reduction in quality open spaces for urban community's recreational needs (Omar, Illia \& Hanita, 2015). Rasidi, Jamirsah and Said (2012) emphasise on the need to maintain quality open spaces as Malaysia is in the quest of providing more urban and suburban landscapes. Hence, several questions are raised in this paper, which are: Why do people need open spaces? What is the type of 
PLANNING MALAYSIA

Journal of the Malaysia Institute of Planners (2017)

interaction they get from visiting open spaces? What are the benefits they get and do these benefits affect their quality of life?

\section{RESEARCH METHODOLOGY}

\section{Theoretical Framework}

Matsuoka and Kaplan (2008) identify major themes that directly linked to the open spaces including human-nature interaction and human-human interaction. The domains then applied in the study as the general guidelines. Under the nature needs, three variables were measured: contact with nature, aesthetic preference, and recreation or play. As for the human needs, the variables measured were social interaction, citizen participation and the sense of community. Table 1 below shows the framework.

Table 1 Theoretical Framework of Human Interaction in Open Spaces

\begin{tabular}{|c|c|c|c|c|c|c|c|}
\hline \multirow[t]{2}{*}{ Author } & \multicolumn{3}{|c|}{ Nature needs } & \multicolumn{3}{|c|}{ Human needs } & \multirow{2}{*}{ 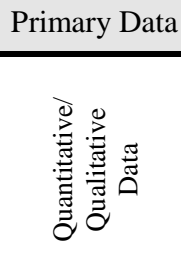 } \\
\hline & 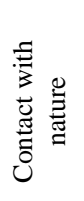 & 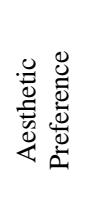 & 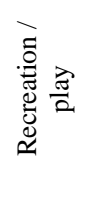 & 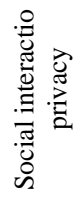 & 荵 & 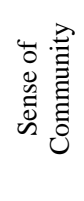 & \\
\hline Austin (2004) & - & & & - & & - & Qualitative \\
\hline Chiesura (2004) & $\bullet$ & & - & $\bullet$ & $\bullet$ & & Quantitative \\
\hline Gobster (2001) & $\bullet$ & $\bullet$ & $\bullet$ & & $\bullet$ & $\bullet$ & Both \\
\hline Oguz (2000) & $\bullet$ & $\bullet$ & $\bullet$ & $\bullet$ & & & Qualitative \\
\hline $\begin{array}{l}\text { Ozguner \& Kendle } \\
\text { (2006) }\end{array}$ & $\bullet$ & $\bullet$ & & $\bullet$ & & & Quantitative \\
\hline Abu-Ghazzeh (1996) & $\bullet$ & & & & $\bullet$ & $\bullet$ & Qualitative \\
\hline Crow et.al (2006) & $\bullet$ & $\bullet$ & $\bullet$ & $\bullet$ & & & Quantitative \\
\hline $\begin{array}{l}\text { Dokmeci \& Berkoz } \\
(2000)\end{array}$ & $\bullet$ & $\bullet$ & & $\bullet$ & & & Quantitative \\
\hline Hull et.al (1994) & $\bullet$ & & & & & $\bullet$ & Qualitative \\
\hline Lucy \& Phillips (1997) & $\bullet$ & & & & & $\bullet$ & Qualitative \\
\hline Vogt \& Marans (2004) & $\bullet$ & $\bullet$ & $\bullet$ & $\bullet$ & & & Qualitative \\
\hline $\begin{array}{l}\text { Herrington \& Studtman } \\
\text { (1998) }\end{array}$ & $\bullet$ & & & $\bullet$ & & & Qualitative \\
\hline Coles \& Bussey (2000) & $\bullet$ & & & $\bullet$ & $\bullet$ & & Both \\
\hline Simson (2000) & $\bullet$ & $\bullet$ & & & & $\bullet$ & Qualitative \\
\hline Yuen \& Hien (2005) & $\bullet$ & $\bullet$ & $\bullet$ & $\bullet$ & & & Qualitative \\
\hline
\end{tabular}

Source: Matsuoka \& Kaplan (2008) 
Filzani Illia Ibrahim, Dasimah Omar, \& Nik Hanita Nik Mohamad

Human Interaction in Urban Spaces: A Quantitative Analysis in Urban Park, Shah Alam City, Selangor

\section{Variables Measured}

This research explores multi-dimensional human needs in open spaces and the perceived benefits from the interactions towards the area. The design of the spatial configuration could serve as a platform for human-nature interaction or human-human interaction. In order to comprehend design qualities that encourages interactions, it is recommended to measure the open spaces properties (i.e. green quality, green setting, accessibility, facilities and amenities) and interactions (human-nature interaction and human-human interaction) of the open space through documented responses. The unit of analysis is the various range of age group of the open spaces of Zone A in Shah Alam, Selangor. The approach in dividing the variables into two major categories was for the purpose of systematic data collection and to gauge how daily usage pattern of open spaces relate to the interactions.

\section{Methods of Data Collection}

A total of 1,000 of survey questionnaires were distributed within the various open spaces in Zone A, Shah Alam, Selangor. Questionnaires were distributed randomly regardless the users' age, race and ethnicity. However, only 861 were completed by respondents. In this survey, respondents were provided with a survey form with subsections to determine their background, such as gender, income, companionship, mode of transportation to open space, frequency of visit and time spend per visit. Respondents also were asked their main purpose of visiting open space. This were divided into two subsections: the human-nature interaction and the human-human interaction.

\section{Study Area}

The selected study area is Zone A, Shah Alam, Selangor Darul Ehsan, Malaysia. Table 2 below shows the ratio and percentage of the survey respondents.

Table 2 Sample size for each open space

\begin{tabular}{lccc}
\hline Study Area & Size (hectares) & Percentage of size & Sample Size \\
\hline Taman Tasik Shah Alam & 43.0 & 66.3 & 428 \\
Section 7 & 9.89 & 15.2 & 134 \\
Section 18 & 6.0 & 9.3 & 149 \\
Section 8 Playground & 4.0 & 6.2 & 100 \\
Section 4 & 2.0 & 3.0 & 50 \\
\hline Total & 64.89 & 100 & 861 \\
\hline
\end{tabular}

\section{Development of the Instruments and Procedures}

The research instrument was developed based on literature analysis as well as the items tested on multi-dimensional human needs. Various useable items from human needs pattern studies were integrated to develop further the methods. Every measurement were structured using 5-level Likert Scale that were 1: Strongly Disagree; 2: Disagree; 3: Neutral, 4: Agree and 5: Strongly Agree. 


\section{RESULTS AND FINDINGS}

The data from survey questionnaires were coded into SPSS software for statistical analyses. The main focused of the analyses was to understand the relationship of human-human interactions and human-nature interactions that took place in the open space area. Demographic attributes such as gender, race, age group and home distance to open space were also considered. The descriptive analysis in Table 3 provides an overall analysis of the respondents profile in the study area.

Table 3 Overall Descriptive Analysis on Profile of Respondents

\begin{tabular}{|c|c|c|c|c|c|c|c|c|c|c|}
\hline \multirow{2}{*}{$\begin{array}{l}\text { Descriptive } \\
\text { Analysis }\end{array}$} & \multicolumn{2}{|c|}{ Section 18} & \multicolumn{2}{|c|}{ Section 8} & \multicolumn{2}{|c|}{ Section 7} & \multicolumn{2}{|c|}{ Section 4} & \multicolumn{2}{|c|}{ Section 2} \\
\hline & $\mathrm{N}$ & $\%$ & $\mathrm{~N}$ & $\%$ & $\mathrm{~N}$ & $\%$ & $\mathrm{~N}$ & $\%$ & $\mathrm{~N}$ & $\%$ \\
\hline \multicolumn{11}{|l|}{ Gender } \\
\hline Male & 73 & 49.0 & 46 & 46.0 & 62 & 46.3 & 15 & 29.4 & 192 & 44.9 \\
\hline Female & 76 & 51.0 & 54 & 54.0 & 72 & 53.7 & 35 & 68.6 & 236 & 55.1 \\
\hline Total & 149 & 100.0 & 100 & 100.0 & 134 & 100.0 & 50 & 98.0 & 428 & 100.0 \\
\hline \multicolumn{11}{|l|}{ Age Group } \\
\hline $13-19$ & 22 & 14.8 & 25 & 25.0 & 11 & 8.2 & 14 & 27.5 & 71 & 16.6 \\
\hline $20-50$ & 85 & 57.0 & 44 & 44.0 & 110 & 82.1 & 28 & 54.9 & 331 & 77.3 \\
\hline $50-60$ & 37 & 24.8 & 26 & 26.0 & 12 & 9.0 & 8 & 15.7 & 22 & 5.1 \\
\hline$>60$ & 5 & 3.4 & 5 & 5.0 & 1 & 0.7 & 0 & 0 & 4 & 0.9 \\
\hline Total & 149 & 100.0 & 100 & 100.0 & 134 & 100.0 & 50 & 98.0 & 428 & 100.0 \\
\hline \multicolumn{11}{|l|}{ Marital Status } \\
\hline Single & 43 & 28.9 & 25 & 25.0 & 99 & 73.9 & 14 & 27.5 & 264 & 61.7 \\
\hline Married & 103 & 69.1 & 71 & 71.0 & 30 & 22.4 & 36 & 70.6 & 161 & 37.6 \\
\hline Divorce & 3 & 2.0 & 4 & 4.0 & 5 & 3.7 & 0 & 0 & 2 & 0.5 \\
\hline Total & 149 & 100.0 & 100 & 100.0 & 134 & 100.0 & 50 & 98.0 & 428 & 100.0 \\
\hline \multicolumn{11}{|l|}{ Race } \\
\hline Malay & 135 & 90.6 & 99 & 99.0 & 118 & 88.1 & 50 & 98.0 & 396 & 92.5 \\
\hline Chinese & 7 & 4.7 & 0 & 0 & 6 & 4.5 & 0 & 0 & 13 & 3.0 \\
\hline Indian & 6 & 4.0 & 1 & 1.0 & 9 & 6.7 & 0 & 0 & 17 & 4.0 \\
\hline others & 1 & 0.7 & 0 & 0 & 1 & 0.7 & 0 & 0 & 2 & 0.5 \\
\hline Total & 149 & 100.0 & 100 & 100.0 & 134 & 100.0 & 50 & 98.0 & 428 & 100.0 \\
\hline \multicolumn{11}{|l|}{ Types of Job } \\
\hline Government & 72 & 48.3 & 52 & 52.0 & 23 & 17.2 & 33 & 64.7 & 74 & 17.3 \\
\hline Private & 34 & 22.8 & 28 & 28.0 & 24 & 17.9 & 3 & 5.9 & 101 & 23.6 \\
\hline Student & 34 & 22.8 & 20 & 20.0 & 84 & 62.7 & 13 & 25.5 & 222 & 51.9 \\
\hline Others & 9 & 6.0 & 0 & 0 & 3 & 2.2 & 1 & 2.0 & 31 & 7.2 \\
\hline Total & 149 & 100.0 & 100 & 100.0 & 134 & 100.0 & 50 & 98.0 & 428 & 100.0 \\
\hline \multicolumn{11}{|c|}{ Neighbourhood Residents } \\
\hline Yes & 108 & 72.5 & 37 & 37.0 & 110 & 82.1 & 25 & 49.0 & 180 & 42.1 \\
\hline No & 41 & 27.5 & 63 & 63.0 & 24 & 17.9 & 25 & 49.0 & 248 & 57.9 \\
\hline Total & 149 & 100.0 & 100 & 100.0 & 134 & 100.0 & 50 & 98.0 & 428 & 100.0 \\
\hline \multicolumn{11}{|l|}{ Origin } \\
\hline Home & 121 & 81.2 & 79 & 79.0 & 111 & 82.8 & 42 & 82.4 & 272 & 63.6 \\
\hline College/School & 18 & 12.1 & 7 & 7.0 & 13 & 9.7 & 1 & 2.0 & 112 & 26.2 \\
\hline Office & 3 & 2.0 & 14 & 14.0 & 9 & 6.7 & 0 & 0 & 23 & 5.4 \\
\hline Others & 7 & 4.7 & 0 & 0 & 1 & 0.7 & 7 & 13.7 & 21 & 4.9 \\
\hline
\end{tabular}


Filzani Illia Ibrahim, Dasimah Omar, \& Nik Hanita Nik Mohamad

Human Interaction in Urban Spaces: A Quantitative Analysis in Urban Park, Shah Alam City, Selangor

\begin{tabular}{lcccccccccc} 
Total & 149 & 100.0 & 100 & 100.0 & 134 & 100.0 & 50 & 98.0 & 428 & 100.0 \\
\hline Distance & & & & & & & & & & \\
\hline$<1 \mathrm{~km}$ & 44 & 29.5 & 3 & 3.0 & 43 & 32.1 & 4 & 7.8 & 43 & 10.0 \\
$1-2 \mathrm{~km}$ & 72 & 48.3 & 62 & 62.0 & 51 & 38.1 & 37 & 72.5 & 100 & 23.4 \\
$2-5 \mathrm{~km}$ & 26 & 17.4 & 34 & 34.0 & 30 & 22.4 & 9 & 17.6 & 133 & 31.1 \\
\hline $55 \mathrm{~km}$ & 7 & 4.7 & 1 & 1.0 & 10 & 7.5 & 0 & 0 & 152 & 35.5 \\
Total & 149 & 100.0 & 100 & 100.0 & 134 & 100.0 & 50 & 98.0 & 428 & 100.0 \\
\hline Mode of Transportation & 110 & 73 & 43 & 43.0 & 23 & 17.2 & 16 & 31.4 & 122 & 28.5 \\
\hline On Foot & 7 & 4.7 & 0 & 0 & 3 & 2.2 & 1 & 2.0 & 19 & 4.4 \\
Public & 8 & 5.4 & 3 & 3.0 & 69 & 51.5 & 3 & 5.9 & 86 & 20.1 \\
Motorcycle & 22 & 14 & 51 & 51.0 & 39 & 29.1 & 30 & 58.8 & 201 & 47.0 \\
Car & 2 & 1.3 & 3 & 3.0 & 0 & 0 & 0 & 0 & 0 & 0 \\
Others & 149 & 100 & 100 & 100.0 & 134 & 100.0 & 50 & 98.0 & 428 & 100.0 \\
Total & & & & & & & & & & \\
\hline & & & & & & & & & & \\
\hline
\end{tabular}

\section{Frequency and Purpose of Visits}

Figure 1 shows the result of total percentage of visit frequency of the respondents to the open spaces per week. Meanwhile, Figure 2 shows the respondents' purpose of visiting the open spaces.

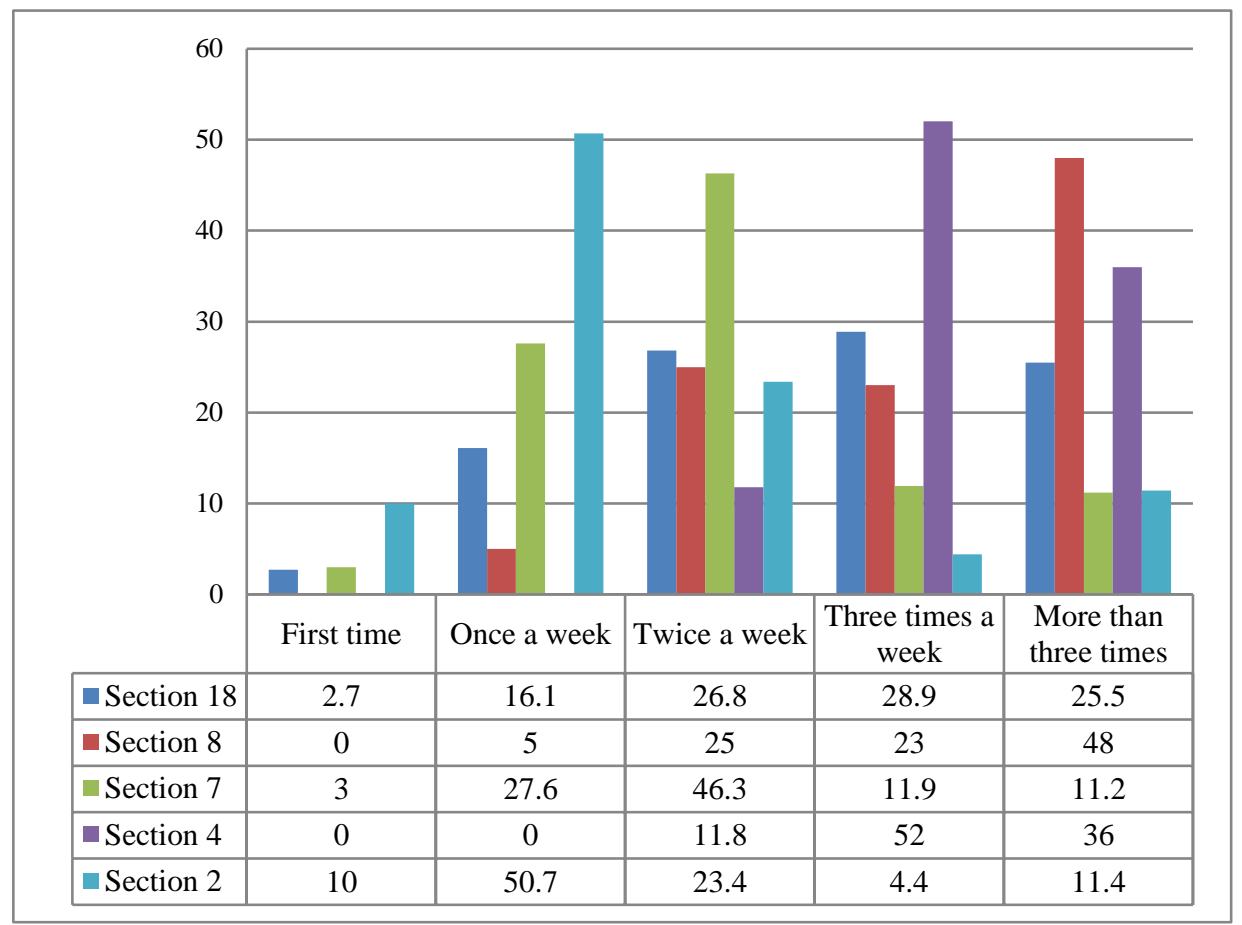

Figure 1 Total Percentage of Visit Frequency 
PLANNING MALAYSIA

Journal of the Malaysia Institute of Planners (2017)

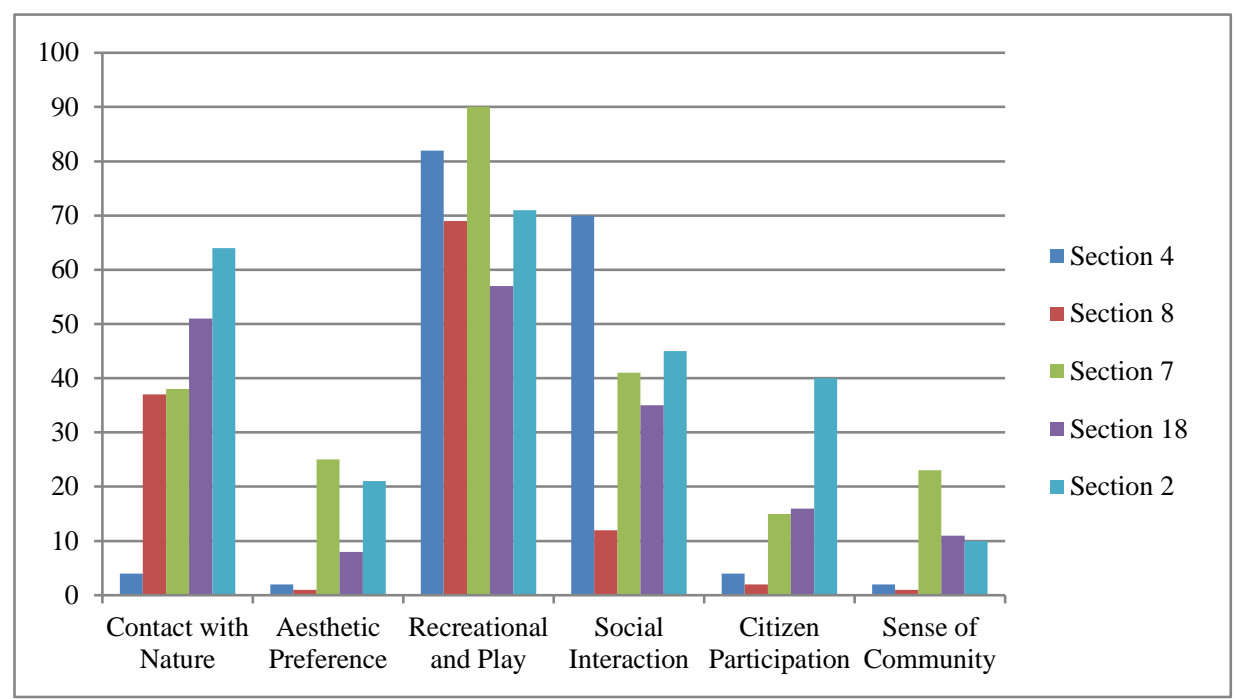

Figure 2 Purpose of Visiting Open Spaces

\section{Human-Nature Interaction}

To explore the human-nature interactions that respondents experienced in the open spaces, the questionnaire were divided into three parts that were contact with nature, aesthetic preference and recreational play. Table 4 below shows the overall results of human-nature interaction.

Table 4 Overall Analysis of Human-Nature Interaction

\begin{tabular}{|c|c|c|c|c|c|c|c|c|c|c|}
\hline \multirow[t]{3}{*}{ Overall Findings } & \multicolumn{10}{|c|}{ Study Areas } \\
\hline & \multicolumn{2}{|c|}{ Section 18} & \multicolumn{2}{|l|}{ Section 8} & \multicolumn{2}{|c|}{ Section 7} & \multicolumn{2}{|l|}{ Section 4} & \multicolumn{2}{|l|}{ Section 2} \\
\hline & $\mathrm{p}$-value & & p-value & & $\mathrm{p}$-value & & p-value & & p-value & \\
\hline \multicolumn{11}{|l|}{ Contact with Nature } \\
\hline Unity with nature & 0.0126 & $\sqrt{ }$ & 0.3443 & $\mathrm{X}$ & 0.1783 & $\mathrm{X}$ & 0.1569 & $\mathrm{X}$ & 0.2440 & $\mathrm{X}$ \\
\hline Unity with my self & 0.2048 & $\mathrm{X}$ & 0.0731 & $\mathrm{X}$ & 0.0765 & $\mathrm{X}$ & 0.7860 & $\mathrm{X}$ & 0.1802 & $\mathrm{X}$ \\
\hline Freedom & 0.0024 & $\sqrt{ }$ & 0.0569 & $\mathrm{X}$ & 0.1990 & $\mathrm{X}$ & 0.7138 & $\mathrm{X}$ & 0.2356 & $\mathrm{X}$ \\
\hline $\begin{array}{l}\text { Recreational } \\
\text { Satisfaction }\end{array}$ & 0.6356 & $\mathrm{X}$ & 0.0985 & $X$ & 0.0412 & $\sqrt{ }$ & 0.7886 & $\mathrm{X}$ & 0.1726 & $\mathrm{X}$ \\
\hline Adventure & 0.1728 & $\mathrm{X}$ & 0.3911 & $\mathrm{X}$ & 0.0095 & $\sqrt{ }$ & 0.0588 & $X$ & 0.7030 & $\mathrm{X}$ \\
\hline Happiness & 0.4380 & $\mathrm{X}$ & 0.0779 & $\mathrm{X}$ & 0.0306 & $\sqrt{ }$ & 0.6805 & $\mathrm{X}$ & 0.3928 & $\mathrm{X}$ \\
\hline $\begin{array}{l}\text { I think open space is } \\
\text { important part of the } \\
\text { city. }\end{array}$ & 0.4594 & $\mathrm{X}$ & 0.0055 & $\sqrt{ }$ & 0.2102 & $\mathrm{X}$ & 0.2295 & $\mathrm{X}$ & 0.6561 & $\mathrm{X}$ \\
\hline \multicolumn{11}{|l|}{ Aesthetic Preference } \\
\hline Unity with nature & 0.1369 & $\mathrm{X}$ & 0.6109 & $\mathrm{X}$ & 0.0807 & $\mathrm{X}$ & 0.7747 & $\mathrm{X}$ & 0.9044 & $\mathrm{X}$ \\
\hline Unity with my self & 0.0377 & $\sqrt{ }$ & 0.7310 & $\mathrm{X}$ & 0.0155 & $\sqrt{ }$ & 0.8888 & $\mathrm{X}$ & 0.1004 & $\mathrm{X}$ \\
\hline Freedom & 0.1750 & $\mathrm{X}$ & 0.8179 & $\mathrm{X}$ & 0.0254 & $\sqrt{ }$ & 0.2529 & $\mathrm{X}$ & 0.1971 & $\mathrm{X}$ \\
\hline $\begin{array}{l}\text { Recreational } \\
\text { Satisfaction }\end{array}$ & 0.1125 & $\mathrm{X}$ & 0.5780 & $X$ & 0.0050 & $\sqrt{ }$ & 0.5740 & $X$ & 0.2315 & $\mathrm{X}$ \\
\hline Adventure & 0.3463 & $\mathrm{X}$ & 0.2680 & $\mathrm{X}$ & 0.0007 & $\sqrt{ }$ & 0.7260 & $\mathrm{X}$ & 0.8400 & $\mathrm{X}$ \\
\hline Happiness & 0.0267 & $\sqrt{ }$ & 0.5099 & $\mathrm{X}$ & 0.0107 & $\sqrt{ }$ & 0.3293 & $\mathrm{X}$ & 0.8873 & $\mathrm{X}$ \\
\hline
\end{tabular}


Filzani Illia Ibrahim, Dasimah Omar, \& Nik Hanita Nik Mohamad

Human Interaction in Urban Spaces: A Quantitative Analysis in Urban Park, Shah Alam City, Selangor

\begin{tabular}{|c|c|c|c|c|c|c|c|c|c|c|}
\hline $\begin{array}{l}\text { I think open space is } \\
\text { important part of the } \\
\text { city. }\end{array}$ & 0.7971 & $\mathrm{X}$ & 0.6512 & $\mathrm{X}$ & 0.2499 & $X$ & 0.6006 & $\mathrm{X}$ & 0.6193 & $\mathrm{X}$ \\
\hline \multicolumn{11}{|l|}{ Recreation and Play } \\
\hline Unity with nature & 0.4405 & $\mathrm{X}$ & 0.1579 & $\mathrm{X}$ & 0.0002 & $\sqrt{ }$ & 0.2953 & $\mathrm{X}$ & 0.0091 & $\sqrt{ }$ \\
\hline Unity with my self & 0.5530 & $\mathrm{X}$ & 0.0857 & $\mathrm{X}$ & 0.0197 & $\sqrt{ }$ & 0.1181 & $\mathrm{X}$ & 0.0087 & $\sqrt{ }$ \\
\hline Freedom & 0.6048 & $\mathrm{X}$ & 0.0886 & $\mathrm{X}$ & 0.0028 & $\sqrt{ }$ & 0.2575 & $\mathrm{X}$ & 0.0649 & $\mathrm{X}$ \\
\hline $\begin{array}{l}\text { Recreational } \\
\text { Satisfaction }\end{array}$ & 0.9955 & $\mathrm{X}$ & 0.0492 & $\sqrt{ }$ & 0.0001 & $\sqrt{ }$ & 0.3626 & $X$ & 0.0351 & $\sqrt{ }$ \\
\hline Adventure & 0.6145 & $\mathrm{X}$ & 0.6607 & $\mathrm{X}$ & 0.0035 & $\sqrt{ }$ & 0.1913 & $\mathrm{X}$ & 0.1434 & $\mathrm{X}$ \\
\hline Happiness & 0.4991 & $\mathrm{X}$ & 0.1774 & $\mathrm{X}$ & 0.0000 & $\sqrt{ }$ & 0.6294 & $\mathrm{X}$ & 0.0634 & $\mathrm{X}$ \\
\hline $\begin{array}{l}\text { I think open space is } \\
\text { important part of the } \\
\text { city. }\end{array}$ & 0.4553 & $\mathrm{X}$ & 0.0605 & $\mathrm{X}$ & 0.0001 & $\sqrt{ }$ & 0.1197 & $X$ & 0.1779 & $X$ \\
\hline
\end{tabular}

\section{Human-Human Interaction}

To determine the human-human interaction that the respondents experienced in the open spaces, the questionnaire were divided into three parts that were social interaction, citizen participation and a sense of community towards the open spaces. Table 5 below shows the overall results.

Table 5 Overall Analysis of Human-Human Interaction

\begin{tabular}{|c|c|c|c|c|c|c|c|c|c|c|}
\hline \multirow[t]{3}{*}{ Overall Findings } & \multicolumn{10}{|c|}{ Study Areas } \\
\hline & \multicolumn{2}{|c|}{ Section 18} & \multicolumn{2}{|l|}{ Section 8} & \multicolumn{2}{|l|}{ Section 7} & \multicolumn{2}{|l|}{ Section 4} & \multicolumn{2}{|l|}{ Section 2} \\
\hline & p-value & & p-value & & p-value & & p-value & & p-value & \\
\hline \multicolumn{11}{|l|}{ Social Interaction } \\
\hline Unity with nature & 0.1277 & $\mathrm{X}$ & 0.2097 & $\mathrm{X}$ & 0.6241 & $\mathrm{X}$ & 0.0149 & $\sqrt{ }$ & 0.0084 & $\sqrt{ }$ \\
\hline Unity with my self & 0.2179 & $\mathrm{X}$ & 0.6136 & $\mathrm{X}$ & 0.6973 & $\mathrm{X}$ & 0.4781 & $\mathrm{X}$ & 0.0117 & $\sqrt{ }$ \\
\hline Freedom & 0.1658 & $X$ & 0.6695 & $\mathrm{X}$ & 0.6568 & $X$ & 0.4208 & $X$ & 0.2127 & $X$ \\
\hline $\begin{array}{l}\text { Recreational } \\
\text { Satisfaction }\end{array}$ & 0.4434 & $X$ & 0.0908 & $\mathrm{X}$ & 0.8026 & $\mathrm{X}$ & 0.7859 & $\mathrm{X}$ & 0.1018 & $X$ \\
\hline Adventure & 0.2254 & $X$ & 0.4801 & $\mathrm{X}$ & 0.8822 & $\mathrm{X}$ & 0.2625 & $\mathrm{X}$ & 0.2125 & $\mathrm{X}$ \\
\hline Happiness & 0.0373 & $\sqrt{ }$ & 0.7493 & $\mathrm{X}$ & 0.7761 & $X$ & 0.7512 & $X$ & 0.0286 & $\sqrt{ }$ \\
\hline $\begin{array}{l}\text { I think open space is } \\
\text { important part of the } \\
\text { city. }\end{array}$ & 0.1206 & $\mathrm{X}$ & 0.4831 & $\mathrm{X}$ & 0.5874 & $\mathrm{X}$ & 0.1931 & $\mathrm{X}$ & 0.1395 & $\mathrm{X}$ \\
\hline \multicolumn{11}{|l|}{ Citizen Participation } \\
\hline Unity with nature & 0.7147 & $\mathrm{X}$ & 0.9540 & $\mathrm{X}$ & 0.0893 & $\mathrm{X}$ & 0.1569 & $\mathrm{X}$ & 0.0176 & $\sqrt{ }$ \\
\hline Unity with my self & 0.7712 & $\mathrm{X}$ & 0.0993 & $\mathrm{X}$ & 0.0977 & $\mathrm{X}$ & 0.3442 & $X$ & 0.0089 & $\sqrt{ }$ \\
\hline Freedom & 0.4831 & $\mathrm{X}$ & 0.9287 & $\mathrm{X}$ & 0.1303 & $\mathrm{X}$ & 0.5347 & $\mathrm{X}$ & 0.0018 & $\sqrt{ }$ \\
\hline $\begin{array}{l}\text { Recreational } \\
\text { Satisfaction }\end{array}$ & 0.2674 & $\mathrm{X}$ & 0.3303 & $\mathrm{X}$ & 0.8605 & $\mathrm{X}$ & 0.7886 & $\mathrm{X}$ & 0.0006 & $\sqrt{ }$ \\
\hline Adventure & 0.9455 & $\mathrm{X}$ & 0.8981 & $\mathrm{X}$ & 0.5659 & $\mathrm{X}$ & 0.5487 & $\mathrm{X}$ & 0.0529 & $\mathrm{X}$ \\
\hline Happiness & 0.6462 & $\mathrm{X}$ & 0.2857 & $\mathrm{X}$ & 0.1068 & $\mathrm{X}$ & 0.8503 & $\mathrm{X}$ & 0.0056 & $\sqrt{ }$ \\
\hline $\begin{array}{l}\text { I think open space is } \\
\text { important part of the } \\
\text { city. }\end{array}$ & 0.0864 & $\mathrm{X}$ & 0.0881 & $\mathrm{X}$ & 0.0868 & $X$ & 0.8793 & $\mathrm{X}$ & 0.0023 & $\sqrt{ }$ \\
\hline \multicolumn{11}{|l|}{ Sense of Community } \\
\hline Unity with nature & 0.4535 & $\mathrm{X}$ & 0.0099 & $\sqrt{ }$ & 0.0826 & $\mathrm{X}$ & 0.0124 & $\sqrt{ }$ & 0.2132 & $\mathrm{X}$ \\
\hline Unity with my self & 0.2936 & $\mathrm{X}$ & 0.0044 & $\sqrt{ }$ & 0.1102 & $X$ & 0.8888 & $X$ & 0.4400 & $\mathrm{X}$ \\
\hline Freedom & 0.1800 & $\mathrm{X}$ & 0.8179 & $\mathrm{X}$ & 0.1609 & $\mathrm{X}$ & 0.7361 & $\mathrm{X}$ & 0.1796 & $\mathrm{X}$ \\
\hline
\end{tabular}


PLANNING MALAYSIA

Journal of the Malaysia Institute of Planners (2017)

\begin{tabular}{|c|c|c|c|c|c|c|c|c|c|c|}
\hline $\begin{array}{l}\text { Recreational } \\
\text { Satisfaction }\end{array}$ & 0.0385 & $\sqrt{ }$ & 0.5780 & $X$ & 0.3750 & $X$ & 0.3941 & $X$ & 0.0803 & $X$ \\
\hline Adventure & 0.9776 & $X$ & 0.6757 & $X$ & 0.1932 & $\mathrm{X}$ & 0.0016 & $\sqrt{ }$ & 0.0882 & $\mathrm{X}$ \\
\hline Happiness & 0.8554 & $X$ & 0.5380 & $X$ & 0.0831 & $\mathrm{X}$ & 0.6688 & $X$ & 0.2955 & $\mathrm{X}$ \\
\hline $\begin{array}{l}\text { I think open space is } \\
\text { important part of the } \\
\text { city. }\end{array}$ & 0.5434 & $X$ & 0.6512 & $X$ & 0.0573 & $X$ & 0.6006 & $\mathrm{X}$ & 0.6786 & $X$ \\
\hline
\end{tabular}

\section{Perceived Benefits of Open Spaces}

For the perceived benefits in relation to the open spaces, the variables that represent the items by overall perception of human-human interaction, overall perception of human-nature interaction and average perceived benefits were created by using the regression model (Figure 3 ) to test whether these variables can significantly affect the perceived benefits. The results are shown in Table 6.

\begin{tabular}{|ll|}
\hline & \multicolumn{1}{c|}{$\mathrm{Y}=\beta_{0+} \beta_{1} \mathrm{X}_{1}+\beta_{2} \mathrm{X}_{2}+\varepsilon$} \\
Where, & \\
$\mathrm{Y}$ & $=$ Perceived Benefit \\
$\mathrm{X}_{1}$ & $=$ Human-nature Interaction \\
$\mathrm{X}_{2}$ & $=$ Human-human Interaction \\
$\varepsilon$ & $=$ error
\end{tabular}

Figure 3 The Proposed Regression Model

Table 6 Overall Analysis for the Proposed Model of Perceived Benefits

\begin{tabular}{|c|c|c|c|c|c|c|c|c|}
\hline \multirow[t]{2}{*}{ Model } & \multicolumn{2}{|c|}{$\begin{array}{l}\text { Unstandardized } \\
\text { Coefficients } \\
\end{array}$} & \multirow{2}{*}{$\begin{array}{l}\text { Standardized } \\
\text { Coefficients } \\
\text { Beta }\end{array}$} & \multirow[t]{2}{*}{ Sig. } & \multicolumn{3}{|c|}{$\begin{array}{l}\text { Dependent Variable: Perceived } \\
\text { Benefit }\end{array}$} & \multirow[t]{2}{*}{$\begin{array}{l}\text { Overall } \\
\text { Result }\end{array}$} \\
\hline & B & $\begin{array}{l}\text { Std. } \\
\text { Error }\end{array}$ & & & R-square & F-statistics & p-value & \\
\hline \multicolumn{9}{|l|}{ Section 18} \\
\hline $1 \quad$ (Constant) & 2.685 & .334 & & .000 & 0.059 & 4.004 & 0.021 & \\
\hline $\begin{array}{l}\text { Human- } \\
\text { nature }\end{array}$ & .148 & .075 & .172 & .051 & & & & $X$ \\
\hline $\begin{array}{l}\text { Human- } \\
\text { human }\end{array}$ & .106 & .065 & .142 & .108 & & & & $X$ \\
\hline \multicolumn{9}{|l|}{ Section 8} \\
\hline $1 \quad$ (Constant) & 2.162 & .426 & & .000 & 0.18 & 10.444 & 0 & \\
\hline $\begin{array}{l}\text { Human- } \\
\text { nature }\end{array}$ & .044 & .091 & .045 & .634 & & & & $\mathrm{X}$ \\
\hline $\begin{array}{l}\text { Human- } \\
\text { human }\end{array}$ & .360 & .083 & .412 & .000 & & & & $\sqrt{ }$ \\
\hline \multicolumn{9}{|l|}{ Section 7} \\
\hline \multirow{3}{*}{$\begin{array}{l}\text { (Constant) } \\
\text { Human- } \\
\text { nature } \\
\text { Human- } \\
\text { human }\end{array}$} & 1.572 & .467 & & .001 & 0.206 & 15.685 & 0 & \\
\hline & .064 & .129 & .049 & .619 & & & & $\mathrm{X}$ \\
\hline & .558 & .130 & .424 & .000 & & & & $\sqrt{ }$ \\
\hline
\end{tabular}


Filzani Illia Ibrahim, Dasimah Omar, \& Nik Hanita Nik Mohamad

Human Interaction in Urban Spaces: A Quantitative Analysis in Urban Park, Shah Alam City, Selangor

Section 4

\begin{tabular}{|c|c|c|c|c|c|c|c|c|c|}
\hline 1 & (Constant) & 3.240 & .473 & & .000 & 0.04 & 0.927 & 0.403 & \\
\hline & $\begin{array}{l}\text { Human- } \\
\text { nature }\end{array}$ & .105 & .105 & .159 & .321 & & & & $X$ \\
\hline & $\begin{array}{l}\text { Human- } \\
\text { human }\end{array}$ & .054 & .117 & .073 & .650 & & & & $X$ \\
\hline & ion 2 & & & & & & & & \\
\hline 1 & (Constant) & 1.262 & .221 & & .000 & 0.301 & 56.474 & 0.000 & \\
\hline & $\begin{array}{l}\text { Human- } \\
\text { nature }\end{array}$ & .418 & .065 & .383 & .000 & & & & $\sqrt{ }$ \\
\hline & $\begin{array}{l}\text { Human- } \\
\text { human }\end{array}$ & .222 & .054 & .246 & .000 & & & & $\sqrt{ }$ \\
\hline
\end{tabular}

\section{CONCLUSION}

In conclusion, this study has shown that human interactions are important to the open spaces as both elements respond well to each other. Apart from that, this study has also indicated that nature and human interactions needs elements of open spaces such as the green spaces, water elements and physical attributes to enhance the interactions between human-human and human-nature.

\section{ACKNOWLEDGEMENTS}

This research is funded by Fundamental Research Grant Scheme (FRGS) from Ministry of Higher Education, Malaysia. The authors would also like to thank all officers of Research Management Institute of Universiti Teknologi MARA, particularly whom in charge for this research grant.

\section{REFERENCES}

Machabee, L. G., Oleson, J. J., \& Kinzig, A. P. (2005). Neighborhood parks uses by Phoenix residents: an exploration of socio demographic differences. In D. Harmon (Ed.), People, Places, and Parks: Proceedings of the 2005 George Wright Society Conference on Parks, Protected Areas, and Cultural Sites. Hancock, Michigan: The George Wright Society.

Matsuoka, R. H., \& Kaplan, R. (2008). People needs in the urban landscape : Analysis of Landscape and Urban Planning contributions. Landscape and Urban Planning, 84(1), 7-19. doi: 10.1016/j.landurbplan.2007.09.009

Mutiara, S., \& Isami, K. (2012). Characteristic of public small park usage in Asia Pacific countries: case study in Jakarta and Yokohama City. Procedia - Social and Behavioral Sciences, 35, 412-419. doi:10.1016/j.sbspro.2012.02.106

Omar, D., Illia, F., \& Hanita, N. (2015). Human interaction in open spaces. Procedia Social and Behavioral Sciences, 201, 352-359. Doi: 10.1016/j.sbspro.2015.08.186

Rasidi, M. H., Jamirsah, N., \& Said, I. (2012). Urban green space design affects urban residents' social interaction. Procedia - Social and Behavioral Sciences, 68, 464480. doi:10.1016/j.sbspro.2012.12.242 